\title{
Escala Gestão do Conhecimento em Equipes: Adaptação para o Brasil
}

\author{
Angela de Fátima Saraiva Freitas ${ }^{1}$ \\ Luciana Mourão ${ }^{1}$ \\ ${ }^{1}$ Universidade Salgado de Oliveira, Niterói, RJ
}

\begin{abstract}
Resumo
A gestão do conhecimento é um tema que tem trazido contribuições para diversas áreas, inclusive para a Psicologia Organizacional. O presente estudo objetivou realizar a adaptação para o Brasil e testar algumas evidências de validade da escala Gestão do Conhecimento em Equipe. Foram seguidas as etapas de adaptação semântica, comparação das versões e validade de conteúdo. A escala foi aplicada por meio eletrônico a 496 trabalhadores (68,8\% mulheres) com vínculo empregatício. Foram realizadas análises fatoriais exploratória e confirmatória. A análise paralela e de consistência teórica determinaram o número de fatores, resultando em uma escala com 32 itens, distribuídos em seis fatores, a saber: criação e aquisição do conhecimento, memória da equipe, utilização do conhecimento, partilha e difusão do conhecimento, recuperação do conhecimento, e catalizadores da gestão do conhecimento. Foram identificadas evidências de validade que possibilitam a utilização da escala em amostras brasileiras. Palavras-chave: equipe; gestão do conhecimento, psicometria
\end{abstract}

\section{Team Knowledge Management Scale: Adaptation to Brazil}

\begin{abstract}
Knowledge management is a theme that has brought contributions to various areas, including Organizational Psychology. The present study aimed to adapt to Brazil and test some evidence of validity of the Team Knowledge Management scale. We followed the steps for semantic adaptation, version comparison, and content validity. The scale was made available online and applied to 496 workers $(68.8 \%$ women) with employee status. Data were analyzed by exploratory and confirmatory factorial analysis. The number of factors was determined by parallel and theoretical consistency analyses, resulting in a scale with 32 items, divided into six factors, namely: knowledge creation and acquisition, team memory, knowledge utilization, knowledge sharing and diffusion, knowledge recovery, and knowledge management catalysts. The results allow us to recommend the use of the scale with Brazilian samples.
\end{abstract}

Keywords: teams; knowledge management; psychometrics

\section{Escala Gestión de Conocimiento en Equipos: Adaptación para Brasil}

\section{Resumen}

La gestión de conocimiento es un tema que ha aportado contribuciones en diversas áreas, incluso en la Psicología Organizacional. El objetivo del presente estudio fue realizar la adaptación para Brasil y testar algunas evidencias de validez de la escala Gestión de Conocimiento en Equipo. Fueron seguidas las etapas de adaptación semántica, comparación de las versiones y validez de contenido. Por medio electrónico, la escala fue aplicada a 496 trabajadores (68,8\% mujeres) con vínculo laboral. También fueron realizados análisis factoriales exploratorios y confirmatorios. Los análisis paralelos y de consistencia teórica determinaron el número de factores, resultando una escala con 32 ítems, distribuidos en seis factores, mencionados a seguir: creación y adquisición de conocimiento, memoria de equipo, utilización de conocimiento, reparto y difusión de conocimiento, recuperación de conocimiento y catalizadores de gestión de conocimiento. Fueron identificadas evidencias de validez que posibilitan la utilización de la escala en muestras brasileñas.

Palabras clave: equipos; gestión de conocimiento; psicometría

\section{Introdução}

O cenário mundial transformou-se aceleradamente, sobretudo, a partir da segunda metade do século $\mathrm{XX}$ e as organizações tiveram que se remodelar para acompanhar essas mudanças. O conhecimento passou a ter sua produção acelerada em decorrência do progresso tecnológico e científico, caracterizando-se como um diferencial (Gilan-Deh \& Chamanzamim, 2016; Jannuzzi, Falsarella, \& Sugahara, 2016), a ponto de tornar-se um dos alvos de gestão no âmbito das organizações.

A expressão Gestão do Conhecimento foi utilizada, pela primeira vez no ano de 1993, por Karl Wiig. De lá para cá, a Gestão do Conhecimento tem sido objeto de interesse por parte da comunidade científica e empresarial. Tal interesse justifica-se pela relação que existe entre a gestão do conhecimento e o desempenho organizacional, numa lógica de criação e manutenção de vantagens competitivas sustentáveis - aspectos 
cruciais no atual contexto de constante mudança, elevada incerteza e crescente competitividade (Corfield \& Paton, 2016).

No processo de gestão do conhecimento as equipes são parte importante, já que é nesse nível que ideias, pensamentos e conhecimentos são compartilhados e integrados (Cardoso \& Peralta, 2011). Nesse sentido, Nonaka e Takeuchi (2008) recomendam que na avaliação de gestão do conhecimento seja considerado o nível de análise de grupos/equipes, por ser o elemento que dá suporte e cria sentidos e significados para o conhecimento. Tal recomendação está de acordo com a literatura da área que pondera que as equipes ocupam um espaço central no cenário organizacional (Puente-Palácios \& Albuquerque, 2014; Santos, Mourão, \& Naiff, 2014). Em vista disso, buscou-se um modelo teórico e uma escala de gestão do conhecimento que considerasse a dimensão das equipes, uma vez que os pesquisadores que se interessam pelos fenômenos grupais necessitam de medidas que façam referência a esse nível de análise (Klein \& Koslowski, 2000).

Nessa lógica, o objetivo do presente estudo foi realizar a adaptação para o Brasil e os testes iniciais de validade psicométrica da escala de Gestão do Conhecimento em Equipes (GCE), desenvolvida por Cardoso e Peralta (2011). Embora já existam escalas de gestão do conhecimento desenvolvidas no Brasil ou adaptadas para amostras brasileiras (Batista, 2016; Brito, Ziviani, Oliveira, \& Christino, 2016; Paes, 2014; Sá, Bento, Ziviani, \& Ferreira, 2013), não foi encontrada nenhuma escala específica para os grupos ou equipes, que é o nível de interesse estabelecido para o presente estudo.

Do ponto de vista conceitual, as definições de gestão do conhecimento (Cardoso, 2003; Stollenwerk, 2001; Wiig, 1993), contam com dois aspectos em comum, a saber: (i) o conhecimento é visto como um processo; e (ii) o conhecimento é considerado como um ativo da organização que interfere em seu desempenho. Nesse sentido, adotou-se no presente estudo a definição de Cardoso (2003), a saber: Gestão do conhecimento diz respeito a criação e desenvolvimento das condições organizacionais internas que catalisam todos os processos relacionados com o conhecimento (criação/aquisição, partilha/difusão, armazenamento, recuperação, utilização, etc), no sentido da consecução dos objetivos da organização.

Embora haja muitas publicações sobre gestão do conhecimento, sete modelos teóricos destacam-se na literatura. As dimensões contempladas por cada um desses modelos são as seguintes: (i) Wiig (1993) - criação, explicitação, uso e transferência; (ii) Leonard-Barton (1995) - aquisição, criação, integração e experimentação; (iii) Gold, Malhota e Segars (2001) - aquisição, conversão, aplicação e proteção; (iv) Stollenwerk (2001) - identificação, captura, seleção e validação, organização e armazenagem, compartilhamento, aplicação e criação; $(v)$ Terra (2001) - aquisição, geração, armazenamento e difusão do conhecimento individual e organizacional; (vi) Cardoso (2003) - criação e aquisição, atribuição de sentido, partilha e difusão, memória organizacional, medição e recuperação; e (vii) Cardoso e Peralta (2011) - criação e aquisição, partilha e difusão, memória da equipe; recuperação e utilização; e catalisadores de gestão do conhecimento.

A análise desses modelos teóricos de gestão do conhecimento evidencia que eles não contemplam as mesmas dimensões, embora haja uma dimensão comum em todos os modelos, ainda que com nomenclaturas distintas, a saber: aquisição e/ou criação do conhecimento. Já as dimensões transferência/difusão/ compartilhamento, uso, aplicação ou experimentação dos conhecimentos estão presentes na maioria deles. Observou-se, contudo, que a maior parte dos autores se refere à gestão do conhecimento sem menção direta ao nível das equipes ou das organizações. A referência ao nível organizacional é feita de forma explícita apenas por Terra (2001) e Cardoso (2003), enquanto o nível das equipes de trabalho está restrito a Cardoso e Peralta (2011), que incluem as equipes tanto na dimensão da memória quanto nos catalisadores de gestão do conhecimento, que faz referência direta aos colegas e líderes. Por esse motivo, optou-se por adotar o modelo de Cardoso e Peralta (2011) em função de ser o único que contempla a dimensão das equipes na gestão do conhecimento.

Considerando a decisão de trabalhar com a gestão do conhecimento que contemplasse informações sobre o papel das equipes, cabe esclarecer que a referida medida não se caracteriza como um fenômeno no nível de análise de equipes, conforme discutido por Puente-Palácios e Borba (2009). Trata-se, portanto, de um fenômeno no nível do indivíduo e que leva em conta a percepção que as pessoas têm sobre a possível influência de chefes e colegas sobre os processos de gestão do conhecimento.

Feita tal consideração e escolhida a escala, a próxima etapa foi identificar os instrumentos de medida da gestão do conhecimento já existentes no Brasil. 
Foram, então, consultadas as bases de dados do Scielo, Capes, Google Scholar e CISCO, sem delimitação temporal, utilizando-se como descritores as palavras/ expressões "escala e/ou instrumento e/ou medida" e "Gestão do Conhecimento" ou seus correspondentes na língua inglesa. Nessa pesquisa foram identificados cinco instrumentos, sendo alguns desenvolvidos para avaliação das práticas de gestão do conhecimento específicas de uma organização ou de um segmento de mercado (Batista, 2016; Brito, Ziviani, Oliveira, \& Christino, 2016; Sá, Bento, Ziviani, \& Ferreira, 2013) e dois instrumentos de avaliação de gestão do conhecimento aplicável a contextos mais amplos (Paes, 2014; Terra, 2001). Contudo, nenhum desses instrumentos encontrados na literatura nacional contemplava o nível das equipes de trabalho, por essa razão, optou-se por buscar na literatura estrangeira escalas que contemplassem tal nível.

$\mathrm{Na}$ pesquisa da literatura estrangeira, foram identificados 13 instrumentos de gestão do conhecimento, mas apenas dois contemplavam o nível de equipes. $\mathrm{O}$ primeiro deles, desenvolvido por Cardoso e Peralta (2011), contém cinco seções, sendo que duas delas focalizam as equipes, especificamente em termos de memória e suporte da equipe (catalisadores da gestão do conhecimento). O segundo instrumento, desenvolvido por Singh e Gupta (2014), apresenta quatro dimensões (criação do conhecimento, retenção do conhecimento, suporte ao conhecimento acionável, e compartilhamento do conhecimento), sendo todas voltadas para o nível das equipes de trabalho.

Entre as duas escalas encontradas na literatura estrangeira optou-se pela de Cardoso e Peralta (2011) em função de ela contemplar dimensões tanto do nível individual quanto dimensões específicas das equipes de trabalho; enquanto a escala proposta por Singh e Gupta (2014) tem suas quatro dimensões voltadas para as equipes. Nesse sentido, a escala de Cardoso e Peralta (2011) apresenta como vantagem a possibilidade de investigar a gestão do conhecimento considerando tanto dimensões do nível do indivíduo (criação e aquisição; partilha e difusão; e recuperação e utilização), quanto dimensões do nível da equipe (memória da equipe e catalisadores da gestão do conhecimento). Além disso, a escala de Cardoso e Peralta (2011) apoia-se em um modelo teórico considerado robusto (por contemplar dimensões relevantes e frequentes na literatura relativa ao processo de gestão do conhecimento) e simultaneamente inovador, por propor dimensões voltadas especificamente para o nível das equipes de trabalho. Diante do exposto, considerou-se que a escala de Cardoso e Peralta (2011) atenderia a muitas pesquisas no contexto brasileiro, não sendo necessária a criação de uma escala específica para o Brasil e sendo possível realizar uma adaptação da referida escala para aplicação em amostras nacionais.

A escala de Cardoso e Peralta (2011) avalia 14 dimensões da gestão do conhecimento no contexto das equipes, agrupadas em cinco seções, que correspondem ao modelo teórico de gestão do conhecimento em equipes apresentado pelos autores. Maiores informações sobre esta escala são apresentadas na seção de Instrumentos.

Vale o registro de que a GCE foi utilizada no estudo de Pinto (2014) sobre relações entre os processos de gestão do conhecimento em equipes, as fases de desenvolvimento grupal e a eficácia grupal. A autora não utilizou as três dimensões da seção catalisadores de gestão do conhecimento, ficando, assim, com 11 dimensões da escala original, sendo cada dimensão considerada como uma escala distinta (conforme possibilidade sinalizada por Cardoso e Peralta, 2011). O estudo de Pinto (2014) apontou que a gestão do conhecimento em equipes atua como mediadora da relação entre desenvolvimento grupal e eficácia grupal e os Alphas encontrados no estudo desta autora variaram entre 0,70 a 0,89 .

\section{Método}

O estudo cumpriu as recomendações da literatura especializada para adaptação e validação de escalas de uma realidade cultural para outra (Borsa, Damásio, \& Bandeira, 2012). A seguir são apresentados os procedimentos de adaptação e os testes iniciais de validade para amostras brasileiras da Escala GCE.

\section{Participantes}

A versão adaptada da escala GCE para a realidade brasileira foi aplicada a uma amostra de conveniência de 496 participantes, atendendo ao número mínimo de cinco participantes por item da escala (Hair, Black, Babin, Anderson, \& Tathan, 2009). Como condição de entrada, as exigências foram de que o respondente tivesse vínculo empregatício em alguma organização, e trabalhasse em equipe há pelo menos um ano e tivesse escolaridade mínima de nível médio.

A exigência do vínculo e tempo de trabalho era necessária para permitir uma avaliação mais consistente dos aspectos de gestão de conhecimento presentes na 
escala, notadamente com relação a fazer parte de uma equipe de trabalho. A exigência de escolaridade mínima foi estabelecida em função da complexidade do tipo de avaliação requerida pelos itens da medida. Como condição de saída considerou-se a existência de mais de 30\% de dados faltantes nas respostas ao instrumento, o que levou à exclusão de seis questionários, totalizando uma amostra de 490 casos válidos.

A amostra apresentou idade situada entre 19 e 71 anos, com média de idade de 42 anos (DP = 10,6), sendo $68,8 \%$ do sexo feminino e $31,2 \%$ do sexo masculino. Com relação à escolaridade, a população pesquisada está distribuída em 5,5\% com ensino médio, $23,5 \%$ com graduação e $71 \%$ com pós-graduação. Nem todos os participantes descreveram o segmento de atuação de sua equipe de trabalho. Dentre os que informaram, predominaram os segmentos de saúde $(24,6 \%)$ e educação $(22,6 \%)$, embora também tivesse equipes de tecnologia/informática $(6,4 \%)$, financeiro/ bancário/contábil (5,9\%) e comércio/vendas (4,2\%). O tempo de trabalho médio foi de 14,4 anos (DP = 14,8), com concentração na faixa entre 2 e 20 anos (67\%). Quanto à região de origem, 85,6\% da amostra é da região Sudeste, estando o restante distribuído entre as outras quatro regiões.

\section{Instrumento}

\section{Escala de Gestão do Conhecimento em Equipes -} GCE

A GCE, desenvolvida em Portugal por Cardoso e Peralta (2011), é composta por 88 itens, distribuídos em 14 dimensões, pertencentes a cinco seções, como descrito na Tabela 1. Os itens são respondidos em escala tipo Likert, variando de 1 (não se aplica ou quase não se aplica) a 5 (aplica-se totalmente ou quase totalmente) (e.g.: "Organizamo-nos em função do conhecimento que possuímos"). Além dos itens da escala, todos os sujeitos da amostra responderam a um questionário sociodemográfico para caracterização da amostra.

A primeira seção da GCE, considerada como elemento impulsionador de todas as demais dimensões, refere-se à criação e aquisição de conhecimento e emerge de fontes internas e externas à equipe. As fontes internas são as condições no ambiente capazes de gerar novos conhecimentos; já as fontes externas são os conhecimentos trazidos por novos trabalhadores, os obtidos nas formações de parcerias e pesquisas externas. A atribuição de sentido ao conhecimento refere-se à compreensão e significado atribuído pelos colaboradores a eventos organizacionais, que variam

Tabela 1.

Seções e dimensões da GCE

\begin{tabular}{|c|c|c|c|}
\hline Seção & Dimensão & $\begin{array}{l}\mathrm{N}^{\mathrm{o}} \text { de } \\
\text { itens }\end{array}$ & Alpha \\
\hline \multirow[t]{3}{*}{ Criação e aquisição de conhecimento } & Criação e aquisição externa & 6 & 0,84 \\
\hline & Criação e aquisição interna & 6 & 0,73 \\
\hline & Atribuição de sentido ao conhecimento & 7 & 0,88 \\
\hline \multirow[t]{2}{*}{ Partilha e difusão do conhecimento } & Partilha e difusão intencional & 8 & 0,85 \\
\hline & Partilha e difusão não intencional & 6 & 0,81 \\
\hline \multirow[t]{3}{*}{ Memória da equipe } & Memória interna e intencional & 6 & 0,80 \\
\hline & Memória interna e tácita & 7 & 0,83 \\
\hline & Memória externa & 6 & 0,79 \\
\hline \multirow[t]{3}{*}{ Recuperação e utilização do conhecimento } & Recuperação controlada & 6 & 0,73 \\
\hline & Recuperação automática & 6 & 0,71 \\
\hline & Utilização do conhecimento & 6 & 0,89 \\
\hline \multirow[t]{3}{*}{ Catalisadores de gestão do conhecimento } & O líder & 6 & 0,94 \\
\hline & Os membros da equipe & 6 & 0,92 \\
\hline & A organização & 6 & 0,84 \\
\hline
\end{tabular}


de acordo com as características de cada equipe (Exemplo de item: "Criamos novos conhecimentos quando trabalhamos juntos").

Em seguida, a GCE aborda a seção partilha e difusão do conhecimento dentro da equipe e pela organização, realizadas de modo intencional ou não. No primeiro modo, o conhecimento, geralmente de natureza explícita, é partilhado e difundido com a prática de ações explícitas e intencionais, enquanto, no modo não intencional, o conhecimento, geralmente de natureza tácita e relacionado à experiência pessoal, é difundido informalmente na interação entre as pessoas (Nonaka \& Takeuchi, 2008). Um exemplo de item dessa dimensão seria: "Conversamos sobre conhecimentos importantes mesmo em encontros ocasionais".

A seção memória grupal refere-se ao armazenamento do conhecimento da equipe, que pode ser orientado para duas direções: memória interna, subdividida em intencional ou tácita, e memória externa (Cardoso, 2003). A memória interna intencional é construída pelas rotinas e procedimentos, produtos desenvolvidos, serviços prestados; enquanto a tácita envolve estratégia, políticas, práticas, teorias de ação, estrutura, ecologia e cultura das equipes. Já a memória externa é constituída pelas interações com as demais equipes da organização e pelas informações sobre a equipe em documentos da organização. (Exemplo de item: "O que sabemos está guardado em documentos, p.ex. bases de dados/catálogos internos/intranet").

Com relação à seção recuperação e utilização do conhecimento, o modelo de Cardoso e Peralta (2011) aponta que podem ser realizadas de forma controlada pela própria equipe, por intermédio de reflexão crítica dos processos grupais que promoveram sucesso e por meio da tecnologia que possibilita acessar informações e conhecimentos contidos em repositórios criados pela organização. Quanto à utilização, tem-se a instrumentalidade relacionada ao desenvolvimento de processos, procedimentos, produtos e serviços. (Exemplo de item: "Quando trabalhamos usamos conhecimentos dos quais não temos consciência no momento").

Por fim, a GCE possui a seção catalizadores da gestão do conhecimento, que se refere à ação dos líderes, dos membros da equipe e da organização como um todo como elementos potencializadores das demais dimensões da GCE. Desse modo, aspectos como cultura orientada para a aprendizagem, incentivo à inovação e comunicação aberta, a visão, o apoio e o comprometimento das lideranças com o processo de gestão do conhecimento podem atuar como dinamizadores dos processos de gestão do conhecimento na organização (Cardoso, 2003). São exemplos de itens dessa dimensão temos: "O nosso líder encoraja-nos a inovar", "Encorajamo-nos uns a outros a inovar".

A versão original da escala de Cardoso e Peralta (2011) apresentou Alpha de Cronbach superior a 0,70 em todas as dimensões (Tabela 1) e as correlações entre os itens foram significativas e superiores a 0,30 . Os itens tiveram carga fatorial igual ou superior a 0,40 .

\section{Procedimentos}

O processo iniciou-se com a adaptação de palavras e expressões de alguns itens para o português brasileiro, feito por uma portuguesa residente no Brasil e por uma pesquisadora brasileira que morou em Portugal por um período de quatro anos. Essas pessoas analisaram o instrumento e verificaram equivalências semântica, idiomáticas, culturais e conceituais, gerando a primeira versão brasileira da escala.

Nessa etapa, foram sugeridas pequenas modificações no que se refere à ortografia de algumas palavras que diferem nos dois países (p. ex. equipas para equipes, projectamos para projetamos) e transformação de voz passiva para ativa (p. ex. "É recompensado o trabalho em equipa" para "O trabalho em equipe é recompensado"). Tal versão foi aplicada a um grupo de oito estudantes de mestrado e doutorado em Psicologia Social, para ajustes de redação dos itens e das instruções.

Dessa aplicação foram acatadas todas as sugestões de troca de palavras por sinônimos mais usuais no Brasil (p. ex. recuperamos por resgatamos, acedemos por conversamos) e substituições de expressões, tais como: temos em conta por utilizamos. Além disso, foram sugeridas alterações na redação de dois itens, a saber: (i) "Assistimos a conferencias/seminários, lemos o que se publica e/ou contratamos especialistas externos à equipa" para "Buscamos conhecimentos produzidos fora da equipe (seminários, conferências, consultorias, leituras)", (ii) "Projectamos uma imagem da nossa equipe quando conversamos com os restantes colaboradores da empresa" para "Quando conversamos com os demais colaboradores da empresa passamos uma imagem da equipe que fazemos parte".

Para validação semântica do instrumento, foi realizado um pré-teste em um grupo de 26 trabalhadores de diversos segmentos produtivos $(88,5 \%$ mulheres, idade média de 43,8 anos, com desvio-padrão de 10,2), todos com experiência mínima de um ano em trabalho em equipe. Dois itens da escala foram indicados como 
de difícil entendimento ("Projetamos uma imagem da nossa equipe quando conversamos com os restantes colaboradores da empresa" e "Quando trabalhamos temos em conta sem querer os valores da equipe") que foram reescritos para possibilitar entendimento ("Quando conversamos com os demais colaboradores da empresa passamos uma imagem da equipe que fazemos parte" e "Os valores da equipe são considerados, automaticamente, quando trabalhamos"). As alterações realizadas na escala são indicadas na Tabela 2.

Após esses procedimentos e a aprovação da pesquisa por um Comitê de Ética em Pesquisa (CAEE 89856218.4.000.5289), a versão final da GCE foi disponibilizada aos respondentes com o apoio de uma ferramenta on-line. $\mathrm{O}$ convite para responder à pesquisa, contendo o link para acesso ao questionário, foi enviado por e-mail e por redes sociais. Os objetivos da pesquisa, a informação do anonimato e as condições para participação (ter no mínimo o ensino médio e estar trabalhando em equipes há pelo menos um ano em alguma organização), foram informados na parte inicial do questionário. Assim, a pessoa deveria concordar com as condições do Termo de Consentimento Livre e Esclarecido (TCLE).

\section{Análise de dados}

Para permitir que a Análise Fatorial Confirmatória (AFC) fosse realizada com uma amostra distinta da que foi utilizada para a Análise Fatorial Exploratória (AFE), a amostra foi separada em duas: uma com 290 casos para a AFE e outra com 200 para a AFC. Como a amostra de 290 participantes ficava abaixo de 5 participantes por item da escala, as análises exploratórias foram realizadas duas vezes, uma com a amostra total (490 casos) e outra com a amostra de 290 participantes. Os valores de eigenvalue, variância explicada, cargas fatoriais e Alpha de Cronbach foram praticamente idênticos para as duas amostras, razão pela qual se optou pela subdivisão amostral para a realização da AFE e da AFC.

Para verificação das propriedades psicométricas da escala, as análises de dados foram feitas com apoio de três softwares: Statistical Package for the Social Science SPSS versão 21.0, Analysis of Moment Structures - AMOS, versão 21.0 e Factor versão 8.0. Todos os itens da escala original foram preservados após o procedimento de adaptação e validação semântica. Inicialmente foi feita a análise dos casos faltosos no banco de dados e, em seguida, a análise do KMO (parâmetro adotado de valores acima de 0,70) e teste de esferecidade de Barttlet (que para aceitação dos pressupostos de análise fatorial deveria ser significativo). Em seguida, foram realizados os testes para avaliar a validade da escala, por meio de análises exploratória e confirmatória.

Nos 490 questionários válidos, foram identificados 23 casos aleatórios de missings ( $0,001 \%$ do banco de dados), que estavam distribuídos sem padrão de regularidade ao longo dos 88 itens, que foram substituídos pela mediana do respectivo item. As análises iniciais revelaram que os dados da amostra atendiam aos pressupostos da análise fatorial - KMO acima de 0,70 e teste significativo para esferecidade de Barttlet (Hair et al., 2009). Foram realizados os testes para avaliar a validade da escala, por meio de análises exploratória e confirmatória.

$\mathrm{Na}$ análise fatorial exploratória, utilizou-se o método de análise Fatoração de Eixo Principal - PAF e o método de rotação foi o oblíquo (direct oblimin). Para definição do número de fatores, foram adotados os critérios de análise paralela e da consistência teórica dos itens, além do critério mínimo de três itens por dimensão, sendo cada um deles com cargas fatoriais iguais ou superiores a 0,32 (Hair et al., 2009). Nos casos de itens que carregaram em dois fatores, somente foram mantidos aqueles cuja diferença entre as cargas fatoriais fosse superior a 0,30 , sendo a permanência do item no fator em que ele tivesse maior carga fatorial e adequação teórica. Foi também realizada correlação de Pearson entre as dimensões resultantes da análise fatorial. O parâmetro adotado para a análise de magnitude foi o de Miles e Shevlin (2001), que consideram intensidade moderada entre 0,30 e 0,49 ou alta igual ou superior a 0,50. Para estimativa da confiabilidade do instrumento foram calculados o Alpha de Cronbach, sendo desejados valores iguais ou superiores a 0,70 .

Em seguida, foi realizada a análise fatorial confirmatória, com utilização do método de estimação Maximuum Likelihood, pois os dados apresentavam normalidade multivariada. A análise de adequação do modelo considerou os seguintes índices de ajustamento (Hair et al., 2009): TLI - Tucker-Lewis Index>0,95; CFI Comparative Fit Index> 0,95; RMSEA - Root Mean Square Error of Approximation $(<0,10)$.

\section{Resultados}

Os resultados obtidos nas análises iniciais revelaram que os dados atendiam aos pressupostos da análise fatorial, com KMO de 0,96 e teste de esferecidade de Barttlet significativo $\left(\chi^{2}=30.693,8 ; p<0,001\right)$. Inicialmente foi testado um modelo unifatorial, e como seria 
Tabela 2.

Itens alterados na escala GCE

\begin{tabular}{|c|c|}
\hline Versão Original (Portugal) & Versão adaptada (Brasil) \\
\hline $\begin{array}{l}\text { Integramos o conhecimento que os novos membros da } \\
\text { equipa trazem do exterior. }\end{array}$ & $\begin{array}{l}\text { Integramos o conhecimento que os novos membros da } \\
\text { equipe trazem de experiências externas à organização. }\end{array}$ \\
\hline $\begin{array}{l}\text { Assistimos a conferências/seminários, lemos o que } \\
\text { se publica e/ou contratamos especialistas externos à } \\
\text { equipa. }\end{array}$ & $\begin{array}{l}\text { Buscamos conhecimentos produzidos fora da equipe } \\
\text { (seminários, conferências, consultorias, leituras). }\end{array}$ \\
\hline $\begin{array}{l}\text { Recolhemos novos dados, informação e conhecimento a } \\
\text { partir da comunicação social (e.g., revistas de divulgação, } \\
\text { televisão). }\end{array}$ & $\begin{array}{l}\text { Recolhemos novos dados, informação e conhecimento } \\
\text { a partir dos meios de comunicação (revistas de } \\
\text { divulgação, televisão, jornais). }\end{array}$ \\
\hline $\begin{array}{l}\text { Recorremos a dados, informação e conhecimento sobre } \\
\text { os nossos clientes externos. }\end{array}$ & $\begin{array}{l}\text { Recorremos a dados, informação e conhecimento sobre } \\
\text { os nossos clientes. }\end{array}$ \\
\hline $\begin{array}{l}\text { Recorremos a várias ferramentas (e.g., intranet, internet) } \\
\text { para localizar o conhecimento que falta à nossa equipa. }\end{array}$ & $\begin{array}{l}\text { Recorremos a ferramentas (p. ex., intranet, internet) } \\
\text { para localizar o conhecimento que falta a nossa equipe. }\end{array}$ \\
\hline $\begin{array}{l}\text { Os membros da equipe partilham o "saber fazer" de } \\
\text { cada um. }\end{array}$ & $\begin{array}{l}\text { Os membros da equipe partilham entre si suas } \\
\text { habilidades. }\end{array}$ \\
\hline $\begin{array}{l}\text { Acedemos a conhecimentos importantes em conversas } \\
\text { ocasionais (e.g., quando nos cruzamos no corredor ou } \\
\text { quando tomamos café). }\end{array}$ & $\begin{array}{l}\text { Conversamos sobre conhecimentos importantes mesmo } \\
\text { em encontros ocasionais (p. ex. quando nos cruzamos } \\
\text { no corredor ou tomamos café). }\end{array}$ \\
\hline $\begin{array}{l}\text { Projectamos uma imagem da nossa equipa quando } \\
\text { conversamos com os restantes colaboradores da } \\
\text { empresa. }\end{array}$ & $\begin{array}{l}\text { Quando conversamos com os demais colaboradores da } \\
\text { empresa passamos uma imagem da equipe que fazemos } \\
\text { parte. }\end{array}$ \\
\hline
\end{tabular}

Recuperamos o conhecimento que fomos organizando e armazenando ao longo do tempo.

Recuperamos modos de pensar e de agir que nos conduziram ao sucesso no passado.

Recuperamos informação/conhecimento que está no site/intranet/manuais da empresa.

Recuperamos soluções que foram validadas e armazenadas por nós no passado.

Recuperamos as melhores práticas desenvolvidas pela nossa empresa.

Quando trabalhamos temos em conta o conhecimento do qual não estamos conscientes no momento.

Executamos tarefas sem pensarmos no conhecimento que estamos a aplicar.

Realizamos uma parte do nosso trabalho com base em conhecimento que surge automaticamente.

Quando trabalhamos temos em conta sem querer os valores da nossa equipe.

O nosso conhecimento vê-se nos nossos produtos/ serviços.

O nosso líder incentiva, por palavras e actos, a partilha de conhecimento e de experiências.

Resgatamos o conhecimento que fomos organizando e armazenando ao longo do tempo.

Resgatamos modos de pensar e agir que nos conduziram ao sucesso no passado.

Resgatamos conhecimento que está no site/intranet/ manuais da empresa.

Resgatamos soluções que foram validadas e armazenadas por nós no passado.

Resgatamos as melhores práticas desenvolvidas pela nossa empresa.

Quando trabalhamos utilizamos conhecimentos dos quais não estamos conscientes no momento.

Executamos tarefas sem pensarmos no conhecimento que estamos aplicando.

Realizamos uma parte do nosso trabalho com base em conhecimento que surge automaticamente ou intuitivo.

Os valores da equipe são considerados, automaticamente, quando trabalhamos.

O nosso conhecimento é demonstrado nos nossos produtos/serviços.

O nosso líder incentiva a partilha de conhecimento e de experiências. 
Tabela 2.

Itens alterados na escala GCE (Continuação)

$$
\text { Versão Original (Portugal) }
$$

O nosso líder encoraja, por palavras e actos, uma comunicação aberta.

Encorajamo-nos mutuamente a inovar.

Incentivamos mutuamente, por palavras e actos, a partilha de conhecimento e de experiências.

Encorajamos, por palavras e actos, uma comunicação aberta.

Domina uma cultura que valoriza o conhecimento.

É recompensado o trabalho em equipa

É recompensada a inovação e a substituição de conhecimento ultrapassado.

É financiado o desenvolvimento dos seus colaboradores (e.g., cursos de formação).

Estimula-se um clima de abertura e de partilha em todas as equipas.

de se esperar pelo referencial teórico apresentado, o ajuste do modelo ficou muito abaixo do aceitável, indicando que a solução de um único fator não é a mais indicada para o construto $\left(\chi^{2}=4.896,3 ; p<0,001 ; \chi^{2} /\right.$ $d f=10,55 ; \mathrm{CFI}=0,54$; $\mathrm{TLI}=0,51$; e RMSEA $=0,140$, com intervalo de confiança entre 0,136-0,143).

A indicação do número de fatores da escala foi definida a partir dos resultados da análise paralela, que sinalizou a existência entre 6 e 7 dimensões. A sétima dimensão teria equivalência de valores do eingenvalue empírico e da média dos eingevalues aleatórios $(2,2)$, o que indicaria que esta pode ser uma dimensão possível, mas não claramente definida como dimensão na matriz de correlações. Por essa razão, foram testadas as estruturas fatoriais com seis e sete dimensões, sendo que esta última não apresentou consistência teórica. A estrutura sugerida para a escala foi, portanto, de seis dimensões, com 35 itens, com cargas fatoriais variando de 0,44 a 0,84 . As seis dimensões explicaram $66,1 \%$ da variância das respostas aos itens. A solução com 35 itens passou por nova análise fatorial para verificar se todos os itens permaneceriam na escala e se a estrutura de seis dimensões era mantida. Tal análise indicou a manutenção das seis dimensões e a retirada de dois itens da dimensão de Aquisição e Criação, a saber: "Recolhemos novos dados informação e conhecimento a partir dos meios de comunicação (revistas de divulgação, televisão, jornais)"; e "Recorremos a ferramentas (ex.: intranet,
Versão adaptada (Brasil)

O nosso líder encoraja uma comunicação aberta.

Encorajamo-nos uns aos outros a inovar.

Incentivamos uns aos outros a partilha de conhecimento e de experiências.

Encorajamos uma comunicação aberta na equipe.

Predomina na empresa uma cultura que valoriza o conhecimento.

O trabalho em equipe é recompensado.

A inovação e a substituição de conhecimento ultrapassado são recompensadas.

A empresa investe no desenvolvimento dos seus colaboradores (cursos e outros).

A empresa estimula um clima de abertura e de partilha em todas as equipes. internet) para localizar o conhecimento que falta a nossa equipe". Tais itens apresentaram carga fatorial abaixo de 0,30 nessa nova configuração da escala. Além disso, a sua retirada melhorou o valor do Alpha de Cronbach de 0,82 para 0,84 e o conteúdo deles não foi considerado imprescindível para a dimensão, uma vez que os outros seis itens conseguiam cobrir satisfatoriamente os processos de aquisição e criação. Assim, a solução da AFE foi de uma escala com 33 itens e seis dimensões.

Os resultados da análise revelaram seis dimensões, com sentido teórico e grau de confiabilidade elevado. O número de itens em cada dimensão variou de 4 a 8, com a seguinte distribuição: Criação e aquisição de conhecimento ( $\alpha=0,84-5$ itens); Partilha e difusão do conhecimento ( $\alpha=0,83-6$ itens); Memória da equipe ( $\alpha=0,85-4$ itens); Recuperação de conhecimento $(\alpha=$ $0,85,4$ itens); e Utilização do conhecimento ( $\alpha=0,92-$ 4 itens). Além dessas cinco dimensões, houve também a dimensão catalisadores da gestão do conhecimento $(\alpha$ $=0,95 ; 8$ itens).

Como evidenciado na Tabela (3), foram identificadas correlações bivariadas entre as dimensões, que de acordo com a classificação de Miles e Shevlin (2001), podem ser consideradas de magnitude fraca (8 correlações), moderada (4) e intensa (3). Merecem destaque as correlações entre a criação e aquisição do conhecimento com duas outras dimensões: utilização do conhecimento $(r=0,51, p<0,01)$; e catalizadores 
da gestão do conhecimento intencional $(r=0,53, p<$ $0,01)$, sendo que esta última também teve correlação de magnitude intensa com a memória da equipe $(r=0,50$, $p<0,01)$.

Finalizada a fase exploratória, foi efetuada a análise fatorial confirmatória por meio de modelagem de equações estruturais utilizando o programa $A$ mos 21.0, obtendo-se os seguintes valore iniciais: $\chi^{2}=1.137,1 ; \chi^{2} /$ $d f=2,53 ; \mathrm{p}<0,001 ; \mathrm{CFI}=0,93 ;$ TLI $=0,92 ;$ e RMSEA $=0,056(0,052-0,060)$. O modelo inicial apresentou indicadores marginalmente satisfatórios. Analisados os valores do modification index, observou-se uma correlação entre os erros elevada para cinco pares de itens. Tais pares pertenciam às mesmas dimensões e tinham conteúdo similar, razões pelas quais se decidiu incluir no modelo tais correlações entre os erros. Os cinco pares de itens correlacionados foram: (i) "Em momentos de descontração, contamos piadas relacionadas com o nosso trabalho" e "Trocamos e-mails e telefonemas com histórias engraçadas que aconteceram no trabalho"; (ii) "Quando trabalhamos usamos conhecimentos dos quais não temos consciência no momento" e "Executamos tarefas sem pensarmos no conhecimento que estamos aplicando"; (iii) "Encorajamo-nos uns aos outros a inovar" e "Incentivamos uns aos outros a partilha de conhecimento e de experiências"; (iv) "Nosso conhecimento torna a equipe mais eficaz" e "Alertamo-nos uns aos outros para o que é importante saber"; e (v) "Encorajamo-nos uns aos outros a inovar" e "Incentivamos uns aos outros a partilha de conhecimento e de experiências".

Também foi feita a opção pela retirada do item "O nosso conhecimento é útil para o nosso trabalho" da dimensão Utilização do conhecimento, em função de o mesmo não atingir a carga fatorial mínima de 0,50 esperada para uma análise fatorial confirmatória (Hair et al.,
2009). Além disso, o ajuste do modelo sem tal item ficou ligeiramente superior. Os valores do modelo antes e após o ajuste foram os seguintes: $\chi^{2}=912,45 / 783,98$; $p<0,001 ; \chi^{2} / d f=1,91 / 1,76 ;$ CFI $=0,96 / 0,97 ;$ TLI $=$ 0,95/0,96; e RMSEA $=0,043 / 0,039(0,039-0,047 / 0,35$ 0,44). A análise do Alpha de Cronbach também se mostrou favorável à retirada de tal item passando de 0,90 para 0,92 . Assim, o modelo final do processo de adaptação da escala GCE resultou em uma escala com seis dimensões e 32 itens no total, com indicadores psicométricos favoráveis tanto na AFE quanto na AFC, conforme apresentado na Tabela 4 e na Figura 1.

\section{Discussão}

O estudo aqui relatado teve por objetivo realizar a adaptação para o Brasil e os testes iniciais de validade psicométrica da escala GCE para o contexto brasileiro. Os autores da escala original, Cardoso e Peralta (2011) dividiam o instrumento em cinco seções temáticas, a saber: Criação e aquisição de conhecimento, Partilha e difusão do conhecimento, Memória da equipe, Recuperação e utilização do conhecimento, Catalisadores de gestão do conhecimento. $\mathrm{Na}$ adaptação da escala para o Brasil, essas cinco seções caracterizaram-se como dimensões, sendo que uma delas - Recuperação e utilização do conhecimento - foi desmembrada em "Recuperação de conhecimento" e "Utilização do conhecimento". Desse modo, os resultados das análises com a amostra brasileira confirmaram parcialmente a estrutura da escala original de Cardoso e Peralta (2011), com bastante proximidade entre o que os autores da escala original chamavam de "seções" do instrumento e o que se denominou na versão brasileira de dimensões.

O fato de a escala de Gestão de Conhecimento na versão portuguesa apresentar um maior nível de

Tabela 3.

Coeficientes das correlações entre as variáveis estudadas

\begin{tabular}{lcccccc}
\hline \multicolumn{1}{c}{ Dimensões } & 1 & 2 & 3 & 4 & 5 & 6 \\
\hline (1) Criação e aquisição do conhecimento & 1 & $0,41^{* *}$ & $0,51^{* *}$ & $0,27^{* *}$ & $-0,10$ & $0,53^{* *}$ \\
(2) Memória da equipe & & 1 & $0,37^{* *}$ & $0,21^{* *}$ & $-0,18$ & $0,50^{* *}$ \\
(3) Utilização do conhecimento & & & 1 & $0,21^{* *}$ & $-0,06$ & $0,48^{* *}$ \\
(4) Partilha e difusão do conhecimento & & & & 1 & $0,10^{*}$ & $0,33^{* *}$ \\
(5) Recuperação do conhecimento & & & & & $-0,08$ \\
(6) Catalizadores da gestão do conhecimento & & & & & & 1 \\
\hline
\end{tabular}

Nota. $* p<0,05 ; * * p<0,01$

Psico-USF, Bragança Paulista, v. 24, n. 3, p. 501-515, jul./set. 2019 
Tabela 4.

Análise Fatorial (Principal Axis Factoring) relacionando itens e fatores extraídos

\begin{tabular}{|c|c|c|c|c|c|c|}
\hline Itens & F1 & F2 & F3 & F4 & F5 & F6 \\
\hline O nosso líder encoraja-nos a inovar. & 0,88 & & & $-0,42$ & $-0,44$ & 0,38 \\
\hline $\begin{array}{l}\text { O nosso líder incentiva a partilha de conhecimento e de } \\
\text { experiências. }\end{array}$ & 0,90 & & & $-0,51$ & $-0,45$ & 0,40 \\
\hline $\begin{array}{l}\text { O nosso líder coordena e organiza reuniões para partilharmos } \\
\text { conhecimento. }\end{array}$ & 0,87 & & & $-0,39$ & $-0,33$ & 0,40 \\
\hline $\begin{array}{l}\text { O nosso líder incentiva a aquisição de conhecimento que a equipe } \\
\text { ainda não possui. }\end{array}$ & 0,91 & & & $-0,39$ & $-0,36$ & 0,41 \\
\hline O nosso líder encoraja uma comunicação aberta. & 0,89 & & & $-0,38$ & $-0,36$ & 0,38 \\
\hline O nosso líder alerta-nos para o que é importante saber. & 0,86 & & & $-0,41$ & $-0,38$ & 0,47 \\
\hline Encorajamo-nos uns aos outros a inovar. & 0,82 & & & $-0,36$ & $-0,50$ & 0,41 \\
\hline Incentivamos uns aos outros a partilha de conhecimento e de & 0,80 & & & $-0,42$ & $-0,50$ & 0,43 \\
\hline
\end{tabular}
experiências.

Quando trabalhamos usamos conhecimentos dos quais não temos consciência no momento.

0,72

Executamos tarefas sem pensarmos no conhecimento que estamos aplicando.

Realizamos uma parte do nosso trabalho com base em conhecimento que surge automaticamente ou intuitivo.

Realizamos uma parte do nosso trabalho de forma intuitiva.

O nosso conhecimento melhora o nosso desempenho.

O nosso conhecimento aumenta a nossa produtividade.

O nosso conhecimento torna a equipe mais eficaz.

Alertamo-nos uns aos outros para o que é importante saber.

Em momentos de descontração, contamos piadas relacionadas com o nosso trabalho.

Trocamos e-mails e telefonemas com histórias engraçadas que aconteceram no trabalho.

No final do dia e nos intervalos, conversamos de forma

descontraída sobre os mais variados assuntos.

Conversamos sobre a nossa equipe quando casualmente nos encontramos.

Em momentos de descontração, os colegas mais antigos contamnos histórias passadas na empresa.

Conversamos sobre conhecimentos importantes mesmo em encontros ocasionais (p. ex.: no corredor ou no café).

O conhecimento de cada um é importante.

Aprendemos com os erros que a nossa equipe comete.

0,37

Aprendemos com base no que vamos criando/desenvolvendo.

0,86

Costumamos propor novas ideias.

Criamos novos conhecimentos quando trabalhamos juntos.

$\begin{array}{lll}\mathbf{- 0 , 8 7} & -0,43 & \\ \mathbf{- 0 , 8 9} & -0,34 & \\ \mathbf{- 0 , 8 7} & -0,41 & 0,35 \\ \mathbf{- 0 , 8 4} & & \\ & & \end{array}$

0,72

\section{0,74}

0,75

0,80

0,66 $-0,33 \quad 0,40$

$$
-0,43 \quad-\mathbf{0 , 7 3}
$$

$-0,36 \quad-\mathbf{0 , 8 2}$ $-0,78$ $-0,74$

$\begin{array}{lll}-0,34 & \mathbf{- 0 , 7 6} & 0,35\end{array}$ 
Tabela 4.

Análise Fatorial (Principal Axis Factoring) relacionando itens e fatores extraídos (Continuação)

\begin{tabular}{|c|c|c|c|c|c|c|}
\hline Itens & $\mathrm{F} 1$ & $\mathrm{~F} 2$ & F3 & F4 & F5 & F6 \\
\hline Temos de desaprender alguns hábitos antigos para fazer melhor. & & & & & $-0,61$ & \\
\hline $\begin{array}{l}\text { Temos documentos que explicam o processo de desenvolvimento } \\
\text { dos nossos produtos/serviços. }\end{array}$ & 0,40 & & & & $-0,31$ & 0,82 \\
\hline $\begin{array}{l}\text { Formalizamos e/ou temos formalizados procedimentos e rotinas } \\
\text { para desenvolver o nosso trabalho. }\end{array}$ & 0,44 & & & & & 0,85 \\
\hline $\begin{array}{l}\text { Temos documentos que descrevem as competências necessárias } \\
\text { para a nossa equipe. }\end{array}$ & 0,43 & & & $-0,33$ & & 0,84 \\
\hline $\begin{array}{l}\text { O que sabemos está guardado em documentos (p. ex.: bases de } \\
\text { dados/catálogos internos/intranet). }\end{array}$ & 0,34 & & & & & 0,81 \\
\hline Eigenvalues empíricos (versão 88 itens) & 35,7 & 4,6 & 3,9 & 3,3 & 3,0 & 2,9 \\
\hline Eigenvalues aleatórios (versão 88 itens) & 2,5 & 2,4 & 2,4 & 2,3 & 2,3 & 2,2 \\
\hline Eigenvalues empiricos (versão 32 itens) & 10,8 & 2,9 & 2,7 & 2,4 & 1,9 & 1,7 \\
\hline Alpha de Cronbach & 0,96 & 0,83 & 0,92 & 0,83 & 0,84 & 0,86 \\
\hline Variância explicada (\%) & 32,80 & 8,80 & 8,20 & 7,20 & 5,80 & 5,20 \\
\hline
\end{tabular}

Nota: F1 = catalizadores da gestão do conhecimento; F2 = recuperação do conhecimento; F3 = utilização do conhecimento; F4 = partilha e difusão do conhecimento; F5 = memória da equipe; F6 = criação e aquisição do conhecimento

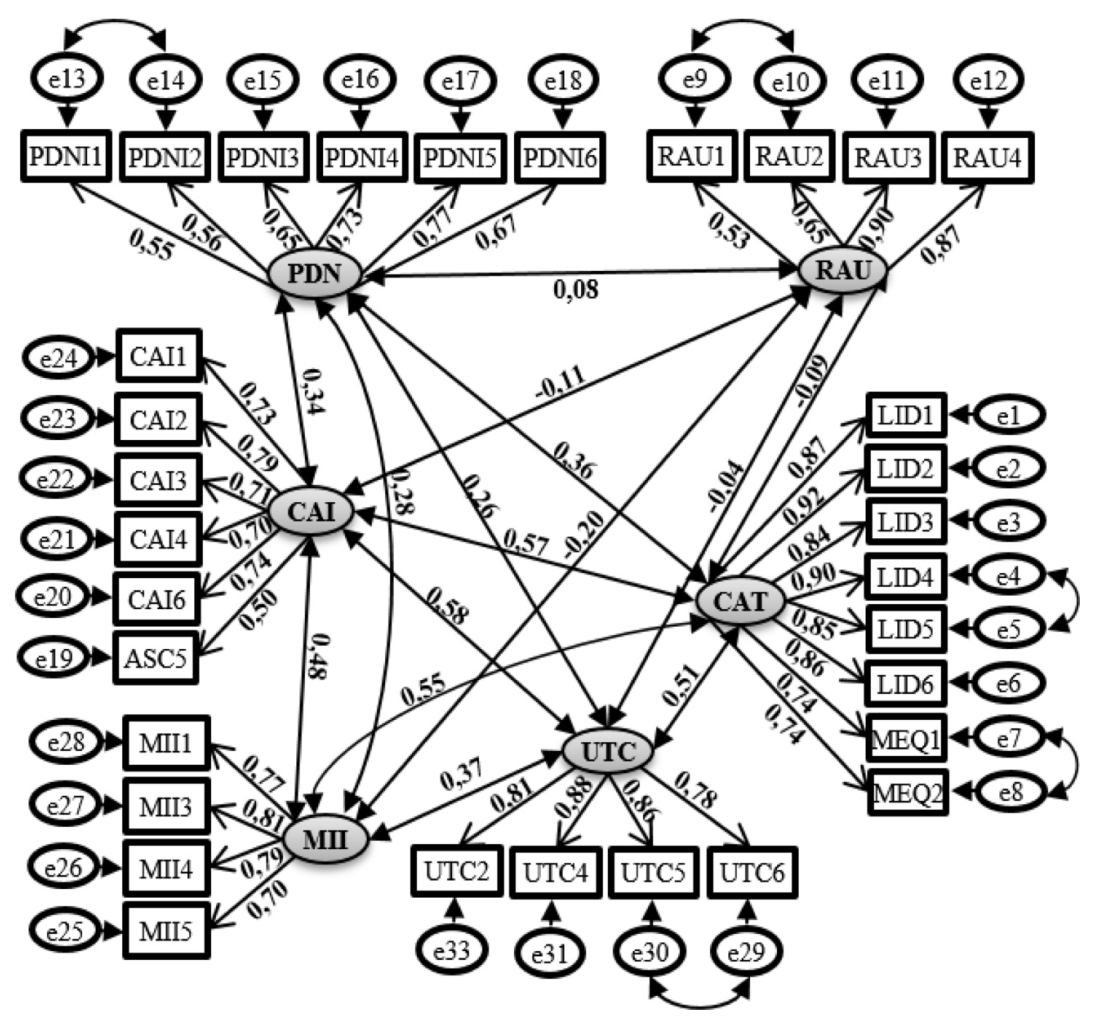

Figura 1. Modelo final da GCE em amostra brasileira

Nota: PDN = Partilha e Difusão do conhecimento; RAU = Recuperação Automática e Utilização; CAI = Criação e Aquisição Intencional; ASC = Atribuição de Sentido ao Conhecimento; MII = Memória Interna Intencional; LID = Líder; MEQ = Membros da Equipe 
detalhamento de seus componentes (cinco seções e 14 dimensões) do que a versão brasileira (seis dimensões), pode estar associado ao fato de que em Portugal a Gestão do Conhecimento possivelmente conte com maior avanço e aplicação nas organizações de trabalho. Nesse sentido, diferenças mais sutis entre os componentes seriam percebidas, o que não aconteceria entre os trabalhadores brasileiros em função de a gestão do conhecimento ainda carecer de maior aplicação no país, por serem temas ainda recentes na história brasileira e no ambiente acadêmico. (Brito et al., 2016). Além disso, o fato de a pesquisa de Cardoso e Peralta (2011) ter sido aplicada em uma única organização e do setor industrial e de a pesquisa brasileira ter sido feita em diferentes organizações e setores também pode ajudar a compreender a diferença na estrutura da escala, pois no estudo em Portugal o contexto era específico, enquanto no estudo no Brasil a amostra era diversificada e com baixa representatividade do setor industrial $(1,7 \%)$.

O desmembramento ocorrido na versão brasileira relativamente à seção do instrumento denominada de Recuperação e utilização do conhecimento está em consonância com os achados de Gilan-Deh e Chamanzamim (2016) que encontraram relação entre a gestão do conhecimento e o comportamento de inovação dos empregados, com dimensões distintas para produção, organização, troca e aplicação do conhecimento. Para tais autores, a recuperação do conhecimento também estaria separada da utilização. Além disso, outros modelos de gestão do conhecimento consideram a aplicação como uma dimensão separada das demais (Gold et al., 2015; Stollenwerk, 2001; Wiig, 1993). Nesse sentido, o resultado encontrado na versão brasileira é corroborado por outros modelos teóricos de gestão do conhecimento e por pesquisas empíricas que apontam a recuperação e utilização como fenômenos distintos.

Apesar de se ter testado também um modelo unifatorial para a gestão do conhecimento, cumpre considerar que tal modelo além de não ser estatisticamente o mais apropriado, também não é o indicado pela literatura da área que considera que os modelos de gestão do conhecimento revelam elementos diferenciados em sua composição, que podem explicar resultados também distintos, por exemplo, em termos de inovação (Gilan-Deh \& Chamanzamim, 2016; Jannuzzi et al., 2016).

Mesmo tendo sido reduzida a quantidade de dimensões e itens, a escala brasileira reflete os elementos básicos presentes no referencial teórico e nos modelos de gestão de conhecimento (Cardoso, 2003; Cardoso \& Peralta, 2011; Stollenwerk, 2001). Na escala original há bastante similitude semântica entre diversos itens de uma mesma dimensão (por exemplo: "Partilhamos experiências e aprendizagens nas reuniões de trabalho" e "Os membros partilham entre si suas habilidades" (Pinto, 2014). Esse aspecto pode ter influenciado a redução elevada de itens da escala.

Algumas dimensões da escala original referiam-se ao mesmo tema, diferenciando-os apenas com relação a alguns critérios, tais como ser externo ou interno, intencional ou não, controlado ou automático, como é o caso da Criação e aquisição do conhecimento, subdividida em três dimensões; Partilha e difusão do conhecimento, subdividida em duas dimensões; Memória da Equipe, subdividida em três dimensões; e Catalisadores de gestão do conhecimento, subdividida em três dimensões. Os itens dessas dimensões se agruparam em um componente mais amplo e muitos deles apresentaram baixa carga fatorial, o que justificou a sua exclusão.

Outro aspecto que merece ser considerado diz respeito à correlação entre as dimensões. $\mathrm{O}$ fato de as correlações entre as dimensões terem sido, em sua maioria, fracas está em consonância com o modelo teórico da GCE, uma vez que ele retrata um processo com etapas sucessivas, nesse sentido, cada uma tem suas características específicas. Mas, se por um lado, correlações pouco intensas seriam o esperado para o construto como um todo; por outro, a dimensão da criação e aquisição do conhecimento é impulsionadora das demais dimensões da gestão do conhecimento, pois emerge de fontes internas da organização que sejam capazes de adquirir, utilizar, compartilhar e armazenar conhecimentos (Cardoso \& Peralta, 2011). Assim, seria de se esperar correlações mais elevadas dessa dimensão com as demais, tal como foi evidenciado nos resultados. Do mesmo modo, há coerência na correlação da dimensão catalizadores da gestão do conhecimento com as demais, em função da sua atuação como potencializadora das outras dimensões da escala GCE.

O fato de a dimensão catalisadores da gestão do conhecimento ter se mantido na versão brasileira da escala é relevante porque tal dimensão diferencia a peculiaridade da medida por voltar-se para fenômenos que ocorrem nas equipes de trabalho. Tal dimensão diz respeito ao papel exercido pela liderança e pelos colegas de equipe (Cardoso \& Peralta, 2011), pois a atuação desses atores impulsiona a criação e aquisição, a utilização, a partilha e o armazenamento do conhecimento.

Do ponto de vista psicométrico, os resultados da AFE apontaram um elevado percentual de variância explicada $(32,8)$ e indicadores satisfatórios de 
confiabilidade da medida, em suas seis dimensões $(\alpha$ $=0,83$ a 0,96). Assim, obteve-se uma boa consistência interna e confiabilidade das dimensões que foram extraídas. A AFC, por sua vez, confirmou a estrutura obtida na AFE, com índices de ajustes satisfatórios.

Para além dos aspectos estatísticos, também é preciso discutir o nível de análise a que se refere o fenômeno. Embora a escala inclua informações sobre o nível de equipes de trabalho, ela foi originalmente construída para uma aplicação e análise no nível individual, ou seja, como os indivíduos percebem os fenômenos de gestão do conhecimento e não uma medida voltada para o nível das equipes. Nesse sentido, a medida não requer uma visão compartilhada pelos membros da equipe acerca do fenômeno na lógica do que é discutido por Puente-Palácios e Borba (2009). Por essa razão, não é feita qualquer discussão em termos de emersão, pois para a aplicação da escala não há uma definição de um fenômeno no nível de análise das equipes. Há tão somente uma análise dos indivíduos sobre a possível atuação dos membros da equipe (chefes e colegas) como catalisadores na gestão do conhecimento.

\section{Considerações finais}

O instrumento ora apresentado fornece evidências de validade que possibilitam a utilização da escala GCE em amostras brasileiras, indicando o seu uso em pesquisas futuras sobre gestão do conhecimento, considerando possíveis contribuições das equipes de trabalho como catalisadores desse processo. Como não havia no Brasil nenhuma escala que considerasse tais catalisadores, este estudo apresenta o primeiro instrumento de verificação da gestão do conhecimento que considera componentes das equipes. Dessa forma, apresenta um potencial de contribuir para os estudos da área, por considerar elementos que não eram previamente analisados.

Assim, a presente pesquisa contribui com a área de Psicologia Organizacional e do Trabalho e áreas afins ao apresentar uma medida que permite mensurar a gestão do conhecimento no nível das equipes de trabalho. Nessa lógica, a escala apresentada neste estudo pode ser utilizada tanto para análises da gestão do conhecimento no âmbito das equipes de diferentes organizações, como para comparação entre equipes de uma mesma organização. O instrumento adaptado permite diagnosticar como está sendo feita a gestão do conhecimento no nível das equipes de trabalho, discriminando as etapas envolvidas nesse processo. Como implicação prática, espera-se que as organizações desenvolvam programas mais eficazes para a gestão do conhecimento em suas respectivas equipes de trabalho.

Cumpre registrar que, apesar da amostra ter sido composta por participantes de todas as regiões do Brasil, foi utilizada uma amostra de conveniência, o que pode ser considerado uma limitação da pesquisa, já que esse fato exige cautela com a universalização dos resultados obtidos. Também deve ser apontada a limitação de terem sido testadas apenas as evidências iniciais de validade da medida.

Estudos futuros poderiam aprofundar os achados atuais. Sugere-se, por exemplo, a aplicação em outras amostras, a investigação da estabilidade temporal da medida e das evidências de validade convergente, concorrente e discriminante. Também seriam úteis estudos com categorias ocupacionais específicas, para identificar como a gestão do conhecimento se manifesta em diferentes segmentos profissionais.

Por fim, considerando-se que as equipes são estruturas muitas vezes necessárias para gerar e partilhar conhecimento, a existência de uma medida que considere a possível contribuição de chefes e colegas pode agregar informações sobre a gestão do conhecimento nas organizações. O uso da escala para mensurar o papel da chefia e dos pares no processo de gestão do conhecimento pode ser útil tanto para um diagnóstico da atuação de tais atores sociais, como também para a proposição de planos de ação que permitam melhorar os processos de criação, aquisição, recuperação, utilização e compartilhamento do conhecimento, com implicações para a memória e o desempenho das equipes de trabalho e dos indivíduos que as compõem.

\section{Referências}

Batista, F. F. (2016). Gestão do conbecimento na administração pública: Resultados da pesquisa IPEA 2014 - niveis de maturidade. Rio de Janeiro: IPEA. Recuperado de http://www.ipea.gov.br/portal/images/stories/ PDFs/TDs/td_2168.pdf

Borsa, J. C., Damásio, B. F., \& Bandeira, D. R. (2012). Adaptação e validação de instrumentos psicológicos entre culturas: Algumas considerações. Paidéia, 22(53), 423-432. doi: 10.1590/1982-43272253201314

Brito, E. M., Ziviani, F., Oliveira, J. L. R., \& Christino, J. M. M. (2016). Gestão do conhecimento no contexto amazônico: Um estudo em cooperativas 
de crédito. Revista Brasileira de Gestão e Desenvolvimento Regional, 12(2), 44-69. Recuperado de http:// www.rbgdr.net/revista/index.php/rbgdr/article/ view/2312/515

Cardoso, L. (2003). Gerir conbecimento e gerar competitividade: Estudo empirico sobre a gestão do conbecimento e seu impacto no desempenho organizacional. (Tese de Doutorado). Universidade de Coimbra, Coimbra, Portugal. Recuperado de https://estudogeral.sib. uc.pt//handle/10316/1014

Cardoso, L., \& Peralta, C. F. (2011). Gestão do conhecimento em equipas: Desenvolvimento de um instrumento de medida multidimensional. Pshychologica, 55, 79-93. Recuperado de http:// impactum-journals.uc.pt/psychologica/article/ view/1122

Corfield, A., \& Paton, R. (2016). Investigating knowledge management: Can KM really change organizational culture? Journal of Knowledge Management, 20(1), 88-103. doi: 10.1108/JKM-12-2014-0502

Gilan-Deh, A. S., \& Chamanzamin, M. R. (2016). Investigating the relationship between knowledge management and employees' innovative behavior at custom organizations of Guilan Province. International Review of Management and Business Research, 5(1), 234-245. Recuperado de http://www.irmbrjournal.com/papers/1456727231.pdf

Gold, A. H., Malhotra, A., \& Segars, A. H. (2015). Knowledge management: An organizational capabilities perspective. Journal of Management Information Systems, 18(1), 185-214. Recuperado de https:// search.proquest.com/docview/218924148?accou ntid $=38384$

Hair, J. F., Black, W. C., Babin, B. J., Anderson, R. E., \& Tathan, R. L. (2009). Análise Multivariada de Dados. Porto Alegre: Bookman.

Jannuzzi, C. S. C., Falsarella, O. M., \& Sugahara, C. R. (2016). Gestão do conhecimento: Um estudo de modelos e sua relação com a inovação nas organizações. Perspectivas em Ciência da Informação, 21(1), 97-118. doi: doi.org/10.1590/1981-5344/2462

Klein, K., \& Kozlowski, S. (2000). Multilevel theory, research and methods in organizations. San Francisco: Jossey-Bass.

Leonard-Barton, D. (1998). Nascentes do Saber: Criando e sustentando as fontes de inovação. Rio de Janeiro: Editora Fundação Getulio Vargas.
Miles, J., \& Shevlin, M. (2001). Applying regression \& correlation: A guide for students and researchs. London: Sage publications.

Nonaka, I., \& Takeuchi, H. (2008). Gestão do conhecimento. Porto Alegre: Bookman.

Paes, L. (2014). Gestão do conhecimento. Em M. M. M. Siqueira (Org.). Novas medidas do comportamento organizacional: Ferramentas de diagnóstico e de gestão (pp.193-208). Porto Alegre: Artmed.

Pinto, A. L. S. (2014). Grupo/equipas de trabalho: Desenvolvimento, gestão do conhecimento e eficácia (Tese de Doutorado). Universidade de Coimbra, Coimbra, Portugal. Recuperado de https://estudogeral.sib. uc.pt/handle/10316/25124

Puente-Palácios, K., \& Albuquerque, F. J. B. (2014). Grupos e equipes de trabalho nas organizações. Em J. C. Zanelli, J. E. Borges-Andrade, \& A. V. B. Bastos (Orgs.), Psicologia, organizações e trabalho no Brasil (pp. 385-412). Porto Alegre: Artmed.

Sá, F. B., Bento, K. G. R., Ziviani, F., \& Ferreira, M. A. T. (2013). Práticas de gestão do conhecimento: Um estudo em organizações mineiras. Perspectivas em Gestão \& Conbecimento, 3(1), 114-131. Recuperado de http://periodicos.ufpb.br/index.php/pgc/ article/view/12259

Santos, A. F. S., Mourão, L., \& Naiff, M. (2014). Representações sociais acerca do trabalho em equipe. Psicologia: Ciência e Profissão, 34(3), 643-659. doi: doi. org/10.1590/S1414-98932008000200012

Singh, R. M., \& Gupta, M. (2014). Knowledge management in teams: Empirical integration and development of a scale. Journal of Knowledge Management, 18(4), 777-794. doi: 10.1108/ JKM-11-2013-0450

Stollenwerk, M. F. L. (2001). Gestão do conhecimento: Conceitos e modelos. Em K. Tarapanoff (Org.). Inteligência organizacional e competitiva (pp. 143-163). Brasília: UnB.

Terra, J. C. C. (2001). Gestão do conhecimento: O grande desafio empresarial. São Paulo: Negócio.

Wiig, K. (1993). Knowledge management foundations: Thinking about thinking - How people and organizations create, represent and use knowledge. Arlington: Schema Press.

Recebido em: 02/04/2018

Reformulado em: 11/09/2018

Aprovado em: 28/09/2018 
Sobre as autoras:

Ângela de Fátima Saraiva Freitas é graduada em Psicologia, mestre e doutoranda em Psicologia pela Universidade Salgado de Oliveira, Niterói, RJ. Professora na Pós-Graduação da AVM Faculdades Integradas e Analista em C\&T na Coordenação de Ensino do Instituto Nacional de Câncer (INCA). Tem particular interesse nos temas desenvolvimento profissional, educação, gestão de pessoas.

ORCID: https://orcid.org/0000-0002- 9318-7841

E-mail: angelafsfreitas@yahoo.com.br

Luciana Mourão é doutora em Psicologia pela Universidade de Brasília. Professora do Programa de Pós-Graduação em Psicologia da Universidade Salgado de Oliveira, Niterói, RJ. Pesquisadora do CNPq. Tem como principais temas de estudo: desenvolvimento profissional, aprendizagem no trabalho e avaliação de programas sociais.

ORCID: https://orcid.org/0000-0002-8230-3763

E-mail:mourao.luciana@gmail.com

Contato com as autoras:

Universidade Salgado de Oliveira

Programa de Pós-graduação em Psicologia

Rua Marechal Deodoro, 217 - Centro

Rio de Janeiro-RJ, Brasil

CEP: 24030-060 